DESY 00-059

hep-ph/0004008

\title{
NLO corrections to the BFKL equation in QCD and in supersymmetric gauge theories.
}

\author{
A.V. Kotikov円 \\ II Institut für Theoretische Physik \\ Universität Hamburg \\ Luruper Chussee 149 \\ 22761 Hamburg, Germany \\ and \\ L.N. Lipatovf \\ St. Petersburg State University and \\ Petersburg Nuclear Physics Institute \\ Gatchina, Orlova Roscha, 188300, St. Petersburg, Russia
}

\begin{abstract}
We study next-to-leading corrections to the integral kernel of the BFKL equation for high energy cross-sections in QCD and in supersymmetric gauge theories. The eigenvalue of the BFKL kernel is calculated in an analytic form as a function of the anomalous dimension $\gamma$ of the local gauge-invariant operators and their conformal spin $n$. For the case of an extended $N=4$ SUSY the kernel is significantly simplified. In particular, the terms non-analytic in $n$ are canceled. We discuss the relation between the DGLAP and BFKL equations in the $N=4$ model.
\end{abstract}

PACS: $12.38 . \mathrm{Bx}$

\footnotetext{
${ }^{1}$ Alexander von Humboldt fellow. On leave of absence from the Particle Physics Laboratory of the Joint Institute for Nuclear Researches, 141980 Dubna, Russia

${ }^{2}$ Supported by the INTAS grant 97-31696 and by the Deutsche Forschungsgemeinschaft
} 


\section{Introduction}

The Balitsky-Fadin-Kuraev-Lipatov (BFKL) equation [1], 2] is used now together with the Dokshitzer-Gribov-Lipatov-Altarelli-Parisi (DGLAP) equation [3] for a theoretical description of structure functions of the deep-inelastic ep scattering at small values of the Bjorken variable $x$. In this kinematical region the structure functions are measured by the $\mathrm{H} 1$ and ZEUS collaborations [4] at DESY. For the DGLAP equation the radiative corrections to the splitting kernels are well known [5]. Although the BFKL equation in the leading logarithmic approximation (LLA) was constructed many years ago, the calculation of the next-to-leading corrections to its kernel was started only in 1989 [6] and completed recently [7, 8]. The total cross-section for the colourless particle scattering is expressed in terms of the solution $G_{\omega}\left(\vec{q}, \overrightarrow{q^{\prime}}\right)$ of the BFKL equation for the $t$-channel partial wave $f_{j}\left(\vec{q} \overrightarrow{q^{\prime}} ; t\right)(j=1+\omega, t=0)$ describing the scattering of the reggeized gluons having their initial and final transverse momenta $\vec{q}$ and $\overrightarrow{q^{\prime}}$ correspondingly.

For unpolarized colourless particle collisions one can average the BFKL kernel over the angle between the two-dimensional vectors $\vec{q}, \overrightarrow{q^{\prime}}$. The eigenvalue of the averaged kernel was calculated in [7]. The high energy behaviour of the total cross-sections $\sigma \sim$ $s^{\Delta}$ is related with the pomeron intercept $\Delta$. It turns out, that in $\overline{M S}$-renormalization scheme the NLO corrections to $\Delta$ are negative and big. However, with the use of nonabelian renormalization schemes and the BLM procedure for the optimal scale setting it was shown [9], that the BFKL predictions for the total cross-section of the process $\gamma^{*} \gamma^{*} \rightarrow$ hadrons turn out to be in an agreement with the experimental data obtained by the L3 collaboration [10]. There are other resummation methods (see [11] and references therein) which lead to quite similar numerical results.

Because the initial virtual photons of the process $\gamma^{*} \gamma^{*} \rightarrow$ hadrons can be polarized, one should know also the NLO corrections to the spin correlators at high energies. It is one of the reasons, why in this paper we calculate next-to-leading corrections to the intercept $\Delta$ of the BFKL pomeron with an arbitrary conformal spin $n$. The case $n=0$ corresponds to the cross-section $\sigma_{0}$ averaged over the angle $\vartheta$ between the photon spins (see [7]) and the case $n=2$ describes the high energy behaviour $s^{\Delta_{2}}$ of the contribution $\sigma_{2}$ proportional to $\cos ^{2} \vartheta-\frac{1}{2}$.

On the other hand, the contribution of higher conformal spins is interesting from a pure theoretical point of view, because it is related to remarkable mathematical properties of the BFKL equation. To begin with, this equation in LLA is invariant under the Möbius transformation of holomorphic impact parameters

$$
\rho_{k} \rightarrow \frac{a \rho_{k}+b}{c \rho_{k}+d}
$$

for arbitrary complex values of $a, b, c$ and $d$ [12]. This invariance allows us to find its solution for arbitrary momentum transfers $q=\sqrt{-t}$ providing that we know the eigenvalues $\Delta(m, \widetilde{m})$ of the kernel for all conformal spins $n$. The conformal weights $m$ and $\widetilde{m}$

are related to the anomalous dimension $\gamma=\frac{1}{2}+i \nu$ of the local operators belonging to a basic series of unitary representations of the Möbius group as follows 


$$
m=\gamma+\frac{n}{2}, \widetilde{m}=\gamma-\frac{n}{2} .
$$

Further, in LLA the homogeneous BFKL equation in the impact parameter representation can be written as the Schrödinger equation

$$
H_{12} \Psi=E_{12} \Psi, \Delta=-\frac{g^{2}}{8 \pi^{2}} \min E_{12},
$$

where $\Delta$ is the BFKL intercept. The pair Hamiltonian $H_{k l}$ for the interaction of two reggeized gluons with their holomorphic coordinates $\rho_{k}, \rho_{l}$ and momenta $p_{k}=i \partial_{k}, p_{l}=i \partial_{l}$ can be presented as a sum of the holomorphic and anti-holomorphic Hamiltonians [13]:

$$
H_{k l}=h_{k l}+h_{k l}^{*}
$$

Here

$$
h_{k l}=\ln \left(p_{k} p_{l}\right)+\frac{1}{p_{k}} \ln \left(\rho_{k l}\right) p_{k}+\frac{1}{p_{l}} \ln \left(\rho_{k l}\right) p_{l}+2 \gamma,
$$

$\gamma$ is the Euler constant and $\rho_{k l}=\rho_{k}-\rho_{l}$.

The holomorphic separability of the Hamiltonian is very important for the solution of the generalized BFKL equation [14 for a composite state of $n$ reggeized gluons:

$$
H \Psi=E \Psi, \Delta_{n}=-\frac{g^{2}}{8 \pi^{2}} E .
$$

The Hamiltonian $H$ in the multi-colour QCD $N_{c} \rightarrow \infty$ can be presented as follows [13]:

$$
H=\frac{1}{2}\left(h+h^{*}\right), h=\sum_{k=1}^{n} h_{k, k+1}, \quad\left[h, h^{*}\right]=0 .
$$

Due to the commutativity of $h$ and $h^{*}$ the function $\Psi$ has the property of the holomorphic factorization 13

$$
\Psi=\sum_{r<s} C_{r s} \psi_{r} \widetilde{\psi}_{s}
$$

where $\psi_{r}$ and $\widetilde{\psi_{s}}$ are solutions of the Schrödinger equations

$$
h \psi_{r}=\varepsilon \psi_{r}, h^{*} \widetilde{\psi}_{s}=\widetilde{\varepsilon} \widetilde{\psi}_{s}, E=\frac{1}{2}(\varepsilon+\widetilde{\varepsilon})
$$

with the same values of energies $\varepsilon$ and $\widetilde{\varepsilon}$ in the holomorphic and anti-holomorphic subspaces correspondingly. The coefficients $C_{r s}$ should be chosen from the requirement of a single-valuedness of $\Psi$ in the two-dimensional impact parameter space.

The holomorphic Hamiltonian $h$ has the integrals of motion $q_{r}(r=1,2 \ldots n)$ [13]

$$
q_{r}=\sum_{i_{1}<i_{2}<\ldots<i_{r}} \rho_{i_{1} i_{2}} \rho_{i_{2} i_{3}} \ldots \rho_{i_{r} i_{1}} p_{i_{1}} p_{i_{2}} \ldots p_{i_{r}}
$$

with the properties

$$
\left[q_{r}, q_{s}\right]=0,\left[q_{r}, h\right]=0 .
$$


Moreover, it coincides with the local Hamiltonian of an integrable Heisenberg spin model 15.

The integrability of the model is related to a duality symmetry [16 of $h$ and $q_{r}$ under the canonical transformation

$$
p_{r} \rightarrow \rho_{r, r+1} \rightarrow p_{r+1}
$$

combined with the transposition of these operators.

In particular, in the case of the compound state of three reggeized gluons, where we can use the conformal anzatz

$$
\Psi_{m, \widetilde{m}}=\left(\frac{\rho_{23}}{\rho_{20} \rho_{30}}\right)^{m}\left(\frac{\rho_{23}^{*}}{\rho_{20}^{*} \rho_{30}^{*}}\right)^{\tilde{m}} \varphi_{m, \tilde{m}}\left(x, x^{*}\right), x=\frac{\rho_{12} \rho_{30}}{\rho_{10} \rho_{32}},
$$

the duality equation for $\varphi_{m, \tilde{m}}\left(x, x^{*}\right)$ can be written in the pseudo-differential form

$$
Q_{m, \tilde{m}} \varphi_{m, \widetilde{m}}=a_{m} a_{\widetilde{m}} \varphi_{m, \widetilde{m}}=\left|\lambda_{m}\right| \varphi_{1-m, 1-\widetilde{m}}, a_{m}=x(1-x)(i \partial)^{1+m} .
$$

Using this equation, new three-gluon states having the pomeron and odderon quantum numbers and the intercept $\Delta=0$ were constructed [17]. Note, that the effective action describing the interaction of reggeized gluons with quarks and gluons was derived in ref. 18.

To investigate a possibility of the holomorphic separability of the BFKL kernel at the next-to-leading approximation in QCD and in extended supersymmetric gauge theories is another reason for the calculation of the $n$-dependence of the pomeron intercept. A presumable integrability of the BFKL dynamics in the $N=4$ supersymmetric field theory at $N_{c} \rightarrow \infty$ could be related to remarkable properties of the renormalization group equations in this model. It is well known, that the $\beta$-function here is zero in all orders of the perturbation theory. Moreover, if we consider conformally invariant evolution equations for matrix elements of the quasi-partonic operators introduced in ref. [19], it turns out [20], that for $N=4$ the pair anomalous dimensions for the supersymmetric twist-2 partonic operators with the Lorentz spin $j$ are proportional to $\psi(j-1)-\psi(1)$. It means, that for $N_{c} \rightarrow \infty$ the corresponding Hamiltonian coincides with the local Hamiltonian for an integrable Heisenberg spin model similar to that obtained for the reggeized gluons (see [13, 15]). The integrability of the evolution equations at the $N=4$ theory in the multi-colour limit is important for a verification of the Maldacena guess 21].

In this paper we extend the analysis of article [2] to the NLO level. Namely, we calculate eigenvalues of the angle-dependent eigenfunctions of the NLO BFKL equation for the $t$-channel partial waves in the framework of QCD and in gauge models with an extended supersymmetry. In the case of QCD this analysis allows us to find main NLO corrections to an angle-depended part of cross-sections. On the basis of the study of gauge models with an extended supersymmetry one can attempt to find a possible source for the violation of the Möbius symmetry, which is important for the calculation of the $t$-dependent BFKL-kernel in QCD at the NLO level.

The article is organized as follows. In Section 2 we review the results of ref. [7], calculate the eigenvalues of the NLO BFKL equation in QCD and discuss the asymptotic 
behaviour of cross-sections at large $s$. Section 3 contains the analysis of the gluino and scalar particle contributions and our results for eigenvalues of the NLO BFKL equation in supersymmetric theories. A summary is given in Conclusion. In Appendix A we discuss the reasons for the appearance of the special function $\operatorname{Ls}_{3}(x)$ in our formulae and in Appendix B we consider the Pomeranchuck singularity in QED and in its supersymmetric extension.

\section{NLO corrections to BFKL kernel in QCD}

To begin with, we review shortly the results of ref.[7], where the QCD radiative corrections to the BFKL integral kernel at $t=0$ were calculated. We discuss only the formulae important for our analysis.

The total cross-section $\sigma(s)$ for the high energy scattering of colourless particles $A, B$ written in terms of their impact factors $\Phi_{i}\left(q_{i}\right)$ and the $t$-channel partial wave $G_{\omega}\left(q, q^{\prime}\right)$ for the gluon-gluon scattering is

$$
\sigma(s)=\int \frac{d^{2} q d^{2} q^{\prime}}{(2 \pi)^{2} q^{2} q^{\prime 2}} \Phi_{A}(q) \Phi_{B}\left(q^{\prime}\right) \int_{a-i \infty}^{a+i \infty} \frac{d \omega}{2 \pi i}\left(\frac{s}{s_{0}}\right)^{\omega} G_{\omega}\left(q, q^{\prime}\right), \quad s_{0}=|q|\left|q^{\prime}\right| .
$$

Here $q$ and $q^{\prime}$ are transverse momenta of virtual gluons and $s=2 p_{A} p_{B}$ is the squared invariant mass for the colliding particle momenta $p_{A}$ and $p_{B}$.

Using the dimensional regularization in the $\overline{M S}$-scheme to remove ultraviolet and infrared divergences in intermediate expressions, the BFKL equation for $G_{\omega}\left(q, q^{\prime}\right)$ can be written in the following form

$$
\omega G_{\omega}\left(q, q_{1}\right)=\delta^{D-2}\left(q-q_{1}\right)+\int d^{D-2} q_{2} K\left(q, q_{2}\right) G_{\omega}\left(q_{2}, q_{1}\right)
$$

where

$$
K\left(q_{1}, q_{2}\right)=2 \omega\left(q_{1}\right) \delta^{D-2}\left(q_{1}-q_{2}\right)+K_{r}\left(q_{1}, q_{2}\right)
$$

and the space-time dimension $D=4+2 \varepsilon$ for $\varepsilon \rightarrow 0$. The gluon Regge trajectory $\omega(q)$ and the integral kernel $K_{r}\left(q_{1}, q_{2}\right)$ related to the real particle production have been calculated in [6], 22], 23], 24] and [25]. The final results (see [0]) can be written in the following form correspondingly for the Regge trajectory

$$
\begin{aligned}
& \omega(q)=-\bar{\alpha}_{\mu}\left(\frac{2}{\varepsilon}+2 \ln \left(\frac{q^{2}}{\mu^{2}}\right)\right)-\bar{\alpha}_{\mu}^{2}\left[\left(\frac{11}{3}-\frac{2}{3} \frac{n_{f}}{N_{c}}\right)\left(\frac{1}{\varepsilon^{2}}-\ln ^{2}\left(\frac{q^{2}}{\mu^{2}}\right)\right)\right. \\
& \left.+\left(\frac{67}{9}-2 \zeta(2)-\frac{10}{9} \frac{n_{f}}{N_{c}}\right)\left(\frac{1}{\varepsilon}+2 \ln \left(\frac{q^{2}}{\mu^{2}}\right)\right)-\frac{404}{27}+2 \zeta(3)+\frac{56}{27} \frac{n_{f}}{N_{c}}\right]
\end{aligned}
$$

\footnotetext{
${ }^{3}$ To simplify equations hereafter (except Appendix B) we omit arrows in the notation of transverse momenta $\vec{q}, \overrightarrow{q^{\prime}}, \overrightarrow{q_{1}}, \overrightarrow{q_{2}}, \ldots$, i.e. in our formulae the momenta $\vec{q}, \overrightarrow{q^{\prime}}, \overrightarrow{q_{1}}, \overrightarrow{q_{2}}, \ldots$ will be represented as $q, q^{\prime}, q_{1}, q_{2}, \ldots$, respectively. Note, however, that the momenta $p_{A}$ and $p_{B}$ are $D$-space momenta.
} 
and for the kernel

$$
\begin{aligned}
& K_{r}\left(q_{1}, q_{2}\right)=\frac{4 \bar{\alpha}_{\mu} \mu^{-2 \varepsilon}}{\pi^{1+\varepsilon} \Gamma(1-\varepsilon)}\left\{\frac{1}{\left(q_{1}-q_{2}\right)^{2}}(1\right. \\
& +\bar{\alpha}_{\mu}\left[\left(\frac{11}{3}-\frac{2}{3} \frac{n_{f}}{N_{c}}\right) \frac{1}{\varepsilon}\left(1-\left(\frac{\left(q_{1}-q_{2}\right)^{2}}{\mu^{2}}\right)^{\varepsilon}\left(1-\zeta(2) \varepsilon^{2}\right)\right)-\ln ^{2}\left(\frac{q_{1}^{2}}{q_{2}^{2}}\right)\right. \\
& \left.\left.+\left(\frac{\left(q_{1}-q_{2}\right)^{2}}{\mu^{2}}\right)^{\varepsilon}\left(\frac{67}{9}-2 \zeta(2)-\frac{10}{9} \frac{n_{f}}{N_{c}}+\varepsilon\left(-\frac{404}{27}+14 \zeta(3)+\frac{56}{27} \frac{n_{f}}{N_{c}}\right)\right)\right]\right) \\
& +\bar{\alpha}_{\mu}\left[\left(1+\frac{n_{f}}{N_{c}^{3}}\right) \frac{3\left(q_{1} q_{2}\right)^{2}-2 q_{1}^{2} q_{2}^{2}}{16 q_{1}^{2} q_{2}^{2}}\left(\frac{2}{q_{1}^{2}}+\frac{2}{q_{2}^{2}}+\left(\frac{1}{q_{2}^{2}}-\frac{1}{q_{1}^{2}}\right) \ln \left(\frac{q_{1}^{2}}{q_{2}^{2}}\right)\right)\right. \\
& -\left(3+\left(1+\frac{n_{f}}{N_{c}^{3}}\right)\left(1-\frac{\left(q_{1}^{2}+q_{2}^{2}\right)^{2}}{8 q_{1}^{2} q_{2}^{2}}-\frac{2 q_{1}^{2} q_{2}^{2}-3 q_{1}^{4}-3 q_{2}^{4}}{16 q_{1}^{4} q_{2}^{4}}\left(q_{1} q_{2}\right)^{2}\right)\right) \int_{0}^{\infty} \frac{d x}{q_{1}^{2}+x q_{2}^{2}} \ln \left|\frac{1+x}{1-x}\right| \\
& +\frac{\left(q_{1}^{2}-q_{2}^{2}\right)}{\left(q_{1}-q_{2}\right)^{2}\left(q_{1}+q_{2}\right)^{2}}\left(\ln \left(\frac{q_{1}^{2}}{q_{2}^{2}}\right) \ln \left(\frac{q_{1}^{2} q_{2}^{2}\left(q_{1}-q_{2}\right)^{4}}{\left(q_{1}^{2}+q_{2}^{2}\right)^{4}}\right)-2 \operatorname{Li}_{2}\left(-\frac{q_{1}^{2}}{q_{2}^{2}}\right)+2 \operatorname{Li}_{2}\left(\frac{q_{1}^{2}}{q_{2}^{2}}\right)\right) \\
& \left.\left.-\left(1-\frac{\left(q_{1}^{2}-q_{2}^{2}\right)}{\left(q_{1}-q_{2}\right)^{2}\left(q_{1}+q_{2}\right)^{2}}\right)\left(\int_{0}^{1}-\int_{1}^{\infty}\right) \frac{d x}{\left(q_{2}-x q_{1}\right)^{2}} \ln \left(\frac{x q_{1}^{2}}{q_{2}^{2}}\right)\right]\right\},
\end{aligned}
$$

where $\operatorname{Li}_{2}(x)$ and $\zeta(n)$ are the Euler dilogarithm and Riemann $\zeta$-functions:

$$
\operatorname{Li}_{2}(x)=-\int_{0}^{x} \frac{d t}{t} \ln (1-t), \quad \zeta(n)=\sum_{k=1}^{\infty} k^{-n} .
$$

Here we introduced also

$$
\bar{\alpha}_{\mu}=\frac{g_{\mu}^{2} N_{c} \Gamma(1-\varepsilon)}{(4 \pi)^{2+\varepsilon}}
$$

for the colour group $S U\left(N_{c}\right)$ and $g_{\mu}$ is the QCD coupling constant fixed at the normalization point $\mu$ in the $\overline{M S}$-scheme.

As it was shown in [2], a complete and orthogonal set of eigenfunctions of the homogeneous BFKL equation in LLA is

$$
G_{n, \gamma}\left(q / q^{\prime}, \theta\right)=\left(\frac{q^{2}}{q^{\prime 2}}\right)^{\gamma-1} e^{i n \theta}
$$

The BFKL kernel in this representation is diagonalized up to the effects related with the running coupling constant $\alpha_{s}\left(q^{2}\right)$ :

$$
\omega=\frac{\alpha_{s}\left(q^{2}\right) N_{c}}{\pi}\left[\chi(n, \gamma)+\delta(n, \gamma) \frac{\alpha_{s}\left(q^{2}\right) N_{c}}{4 \pi}\right] .
$$

The calculation of the correction $\delta(n, \gamma)$ for all conformal spins is one of main results of our paper. 


\subsection{The technique of calculations}

The integration over the angle $\theta$ of the transverse gluon momentum has the form

$$
\int_{-\pi}^{\pi} d \theta e^{i n \theta}=2 \int_{0}^{\pi} d \theta \cos (n \theta)=2 \int_{0}^{\pi} d \theta \mathrm{T}_{n}(\cos \theta)
$$

where $\mathrm{T}_{n}(\cos \theta)$ are the Chebyshev polynomials (below $n$ is a positive integer number).

In the framework of the dimensional regularization a natural extension of the Chebyshev polynomial is the Gegenbauer polynomial $\mathrm{C}_{n}^{\lambda}(\cos \theta)$ (see [26]-29]) with the index $\lambda$ related to the transverse space dimension $D-2$ as $\lambda=(D-2) / 2-1=D / 2-2$. When $\lambda=0$ we return to the Chebyshev polynomials because

$$
\mathrm{C}_{n}^{0}(\cos \theta) \equiv \lim _{\lambda \rightarrow 0} \frac{1}{\lambda} \mathrm{C}_{n}^{\lambda}(\cos \theta)=\frac{2}{n} \mathrm{~T}_{n}(\cos \theta) .
$$

To calculate the terms singular at $\varepsilon \rightarrow 0$ it is convenient to to use the formulae from [27, 29] for the traceless products

$$
x^{\left(\mu_{1}, \ldots, \mu_{n}\right)}=\sum_{p \geq 0}^{[n / 2]} \frac{n !(-1)^{p} \Gamma(n-p+\lambda)}{2^{2 p} p !(n-2 p) ! \Gamma(n+\lambda)} g^{\mu_{1} \mu_{2}} \ldots g^{\mu_{2 p-1} \mu_{2 p}} x^{2 p} x^{\mu_{2 p+1}} \ldots x^{\mu_{n}} .
$$

The traceless products are related to the Gegenbauer polynomials $\mathrm{C}_{n}^{\lambda}(\cos \theta)$ as follows

$$
\frac{n}{2} \frac{1}{\lambda} \mathrm{C}_{n}^{\lambda}\left(\hat{q}_{1} \hat{q}_{2}\right)=S_{n}^{(1)}(\lambda) \frac{q_{1}^{\left(\mu_{1}, \ldots, \mu_{n}\right)} q_{2}^{\left(\mu_{1}, \ldots, \mu_{n}\right)}}{\left(q_{1}^{2} q_{2}^{2}\right)^{n / 2}}
$$

where the symbols $\hat{q}_{1}$ and $\hat{q}_{2}$ are the unit vectors parallel to the transverse momenta $q_{1}$ and $q_{2}$,

$$
\hat{q}_{1} \hat{q}_{2}=\frac{\left(q_{1} q_{2}\right)}{\left(q_{1}^{2} q_{2}^{2}\right)^{1 / 2}}
$$

and $S_{n}^{(1)}(\lambda)$ is the following factor

$$
S_{n}^{(1)}(\lambda)=\frac{2^{n-1} \Gamma(n+\lambda)}{\Gamma(n) \Gamma(1+\lambda)}
$$

After the integration over the momentum $q_{2}$ in Eq.(16) we obtain the product of the same vectors 27, 29]

$$
\frac{q_{1}^{\left(\mu_{1}, \ldots, \mu_{n}\right)} q_{1}^{\left(\mu_{1}, \ldots, \mu_{n}\right)}}{q_{1}^{2 n}}=\frac{\Gamma(\lambda) \Gamma(n+2 \lambda)}{2^{n} \Gamma(2 \lambda) \Gamma(n+\lambda)}=S_{n}^{(2)}(\lambda)
$$

Then in the front of the expressions, obtained from the contributions singular at $\varepsilon \rightarrow 0$, the following coefficient

$$
S_{n}(\lambda)=S_{n}^{(1)}(\lambda) S_{n}^{(2)}(\lambda)=\frac{\Gamma(n+2 \lambda)}{\Gamma(n) \Gamma(1+2 \lambda)}
$$


appears. Because $S_{n}(\lambda)$ is factorized and $S_{n}(0)=1$, it does not have any influence on our results. Thus, the contributions singular at $\varepsilon \rightarrow 0$ can be calculated easily with the use of the formulae from [27, 29].

The $\varepsilon$-independent terms are obtained in a similar way, because one can use of the well known formulae (see [29]) to calculate the contributions of the Feynman diagrams containing $\theta$-functions

$$
\theta(y)= \begin{cases}1, & \text { if } y \geq 0 \\ 0, & \text { if } y<0\end{cases}
$$

in the integrands. These $\theta$-functions come from an expansion of the expressions $\left(q_{1}+q_{2}\right)^{-2},\left(q_{1}-x q_{2}\right)^{-2}$ and $\ln \left(q_{1}-q_{2}\right)^{2}$ in the r.h.s. of eq.(19) (see eq.(24), for example).

However, it is more convenient to work directly with the expansion of propagators over the Chebyshev polynomials and to take initially integrals over the angle in the r.h.s. of eq.(19). The useful formulae for the expansion of propagators and the properties of the Chebyshev polynomials are given below

$$
\begin{aligned}
& \frac{q_{1}^{2}-q_{2}^{2}}{\left(q_{1}-q_{2}\right)^{2}}=\left[1+2 \sum_{n=1}^{\infty} \mathrm{T}_{n}\left(\hat{q}_{1} \hat{q}_{2}\right)\left(\frac{q_{1}^{2}}{q_{2}^{2}}\right)^{n / 2}\right] \theta\left(q_{2}^{2}-q_{1}^{2}\right)-\left[q_{1} \leftrightarrow q_{2}\right] \theta\left(q_{1}^{2}-q_{2}^{2}\right) \\
& \ln \left(q_{1}-q_{2}\right)^{2}=\left[\ln q_{2}^{2}-2 \sum_{n=1}^{\infty} \frac{1}{n} \mathrm{~T}_{n}\left(\hat{q}_{1} \hat{q}_{2}\right)\left(\frac{q_{1}^{2}}{q_{2}^{2}}\right)^{n / 2}\right] \theta\left(q_{2}^{2}-q_{1}^{2}\right)+\left[q_{1} \leftrightarrow q_{2}\right] \theta\left(q_{1}^{2}-q_{2}^{2}\right) \\
& 2 \mathrm{~T}_{n}(x) \mathrm{T}_{m}(x)=\mathrm{T}_{n+m}(x)+\mathrm{T}_{n-m}(x) \quad(n \geq m) \\
& \mathrm{T}_{n}(1)=1, \quad \mathrm{~T}_{0}(x)=1, \quad \mathrm{~T}_{n}(-x)=(-1)^{n} \mathrm{~T}_{n}(x) \\
& \mathrm{T}_{2 n}(0)=(-1)^{n}, \quad \mathrm{~T}_{2 n+1}(0)=0
\end{aligned}
$$

\subsection{The results of calculations}

Applying formulae of the previous subsection to eqs.(16)-(19), we obtain the following results for eigenvalues (23):

$$
\begin{aligned}
\chi(n, \gamma) & =2 \Psi(1)-\Psi\left(\gamma+\frac{n}{2}\right)-\Psi\left(1-\gamma+\frac{n}{2}\right) \\
\delta(n, \gamma) & =-\left[\left(\frac{11}{3}-\frac{2}{3} \frac{n_{f}}{N_{c}}\right) \frac{1}{2}\left(\chi^{2}(n, \gamma)-\Psi^{\prime}\left(\gamma+\frac{n}{2}\right)+\Psi^{\prime}\left(1-\gamma+\frac{n}{2}\right)\right)\right. \\
& -\left(\frac{67}{9}-2 \zeta(2)-\frac{10}{9} \frac{n_{f}}{N_{c}}\right) \chi(n, \gamma)-6 \zeta(3) \\
& +\frac{\pi^{2} \cos (\pi \gamma)}{\sin ^{2}(\pi \gamma)(1-2 \gamma)}\left\{\left(3+\left(1+\frac{n_{f}}{N_{c}^{3}}\right) \frac{2+3 \gamma(1-\gamma)}{(3-2 \gamma)(1+2 \gamma)}\right) \cdot \delta_{n}^{0}\right. \\
& \left.-\left(1+\frac{n_{f}}{N_{c}^{3}}\right) \frac{\gamma(1-\gamma)}{2(3-2 \gamma)(1+2 \gamma)} \cdot \delta_{n}^{2}\right\} \\
& \left.-\Psi^{\prime \prime}\left(\gamma+\frac{n}{2}\right)-\Psi^{\prime \prime}\left(1-\gamma+\frac{n}{2}\right)+2 \Phi(n, \gamma)+2 \Phi(n, 1-\gamma)\right],
\end{aligned}
$$


where $\delta_{n}^{m}$ is the Kroneker symbol, and $\Psi(z), \Psi^{\prime}(z)$ and $\Psi^{\prime \prime}(z)$ are the Euler $\Psi$-function and its derivatives. The function $\Phi(n, \gamma)$ is given below

$$
\begin{aligned}
& \Phi(n, \gamma)=-\int_{0}^{1} d x \frac{x^{\gamma-1+n / 2}}{1+x}\left[\frac{1}{2}\left(\Psi^{\prime}\left(\frac{n+1}{2}\right)-\zeta(2)\right)+\operatorname{Li}_{2}(-x)+\operatorname{Li}_{2}(x)\right. \\
&+\ln (x)\left(\Psi(n+1)-\Psi(1)+\ln (1+x)+\sum_{k=1}^{\infty} \frac{(-x)^{k}}{k+n}\right) \\
&\left.+\sum_{k=1}^{\infty} \frac{x^{k}}{(k+n)^{2}}\left(1-(-1)^{k}\right)\right] \\
&=\sum_{k=0}^{\infty} \frac{(-1)^{k}}{k+\gamma+n / 2}\left[\Psi^{\prime}(k+n+1)-\Psi^{\prime}(k+1)+(-1)^{k}\left(\beta^{\prime}(k+n+1)+\beta^{\prime}(k+1)\right)\right) \\
&\left.\quad-\frac{1}{k+\gamma+n / 2}(\Psi(k+n+1)-\Psi(k+1))\right]
\end{aligned}
$$

and

$$
\beta^{\prime}(z)=\frac{1}{4}\left[\Psi^{\prime}\left(\frac{z+1}{2}\right)-\Psi^{\prime}\left(\frac{z}{2}\right)\right]
$$

For the case $n=0$ the results (27) and (28) coincide with ones obtained in [7].

Almost all terms in the right hand side of eq.(27) except the contribution

$$
\tilde{\Delta}(n, \gamma)=\left(\frac{11}{3}-\frac{2}{3} \frac{n_{f}}{N_{c}}\right) \frac{1}{2}\left(\Psi^{\prime}\left(\gamma+\frac{n}{2}\right)-\Psi^{\prime}\left(1-\gamma+\frac{n}{2}\right)\right)
$$

are symmetric under the transformation $\gamma \leftrightarrow 1-\gamma$. Moreover, as it was mentioned in [7], it is possible to cancel $\tilde{\Delta}(n, \gamma)$ if one would redefine the function $q^{\gamma-1}$ by including in it the logarithmic factor

$$
\left(\frac{\alpha_{s}\left(q^{2}\right)}{\alpha_{s}\left(\mu^{2}\right)}\right)^{1 / 2}
$$

Note also, that the term

$$
\left(\frac{67}{9}-2 \zeta(2)-\frac{10}{9} \frac{n_{f}}{N_{c}}\right) \chi(n, \gamma)=\kappa \chi(n, \gamma)
$$

is proportional to the LO contribution. It appears often in the $\overline{M S}$-scheme for the processes involving soft gluons and it is absent in the gluon-bremsstrahlung (GB) scheme [30 being more natural for these processes. In the GB-scheme the term (29) can be incorporated to a new coupling constant $\tilde{\alpha}_{s}\left(q^{2}\right)$ as

$$
\left.\tilde{\alpha}_{s}\left(q^{2}\right)=\alpha_{s}\left(q^{2} e^{-\kappa N_{c} / \beta_{0}}\right)\right) \quad\left(\beta_{0}=\frac{11}{3} N_{c}-\frac{2}{3} n_{f}\right)
$$

and eq.(23) is replaced by

$$
\omega=\frac{\tilde{\alpha}_{s}\left(q^{2}\right) N_{c}}{\pi}\left[\chi(n, \gamma)+\tilde{\delta}(n, \gamma) \frac{\tilde{\alpha}_{s}\left(q^{2}\right) N_{c}}{4 \pi}\right],
$$


where

$$
\begin{aligned}
\tilde{\delta}(n, \gamma) & =-\left[\left(\frac{11}{3}-\frac{2}{3} \frac{n_{f}}{N_{c}}\right) \frac{1}{2}\left(\chi^{2}(n, \gamma)-\Psi^{\prime}\left(\gamma+\frac{n}{2}\right)+\Psi^{\prime}\left(1-\gamma+\frac{n}{2}\right)\right)\right. \\
& -6 \zeta(3)+\frac{\pi^{2} \cos (\pi \gamma)}{\sin ^{2}(\pi \gamma)(1-2 \gamma)}\left\{\left(3+\left(1+\frac{n_{f}}{N_{c}^{3}}\right) \frac{2+3 \gamma(1-\gamma)}{(3-2 \gamma)(1+2 \gamma)}\right) \cdot \delta_{n}^{0}\right. \\
& \left.-\left(1+\frac{n_{f}}{N_{c}^{3}}\right) \frac{\gamma(1-\gamma)}{2(3-2 \gamma)(1+2 \gamma)} \cdot \delta_{n}^{2}\right\} \\
& \left.-\Psi^{\prime \prime}\left(\gamma+\frac{n}{2}\right)-\Psi^{\prime \prime}\left(1-\gamma+\frac{n}{2}\right)+2 \Phi(n, \gamma)+2 \Phi(n, 1-\gamma)\right] .
\end{aligned}
$$

Analogously to ref. [7] we can obtain the eigenvalues (23) in the case of a non-symmetric choice of the normalization $s_{0}$ of the energy in eq.(15). For example, for the scale $s_{0}=q^{2}$, which is natural for deep-inelastic scattering process, we have

in $\overline{M S}$-scheme

$$
\omega=\frac{\alpha_{s}\left(q^{2}\right) N_{c}}{\pi}\left[\chi(n, \gamma)+\left(\delta(n, \gamma)-2 \chi(n, \gamma) \chi^{\prime}(n, \gamma)\right) \frac{\alpha_{s}\left(q^{2}\right) N_{c}}{4 \pi}\right]
$$

and in GB-scheme

$$
\omega=\frac{\tilde{\alpha}_{s}\left(q^{2}\right) N_{c}}{\pi}\left[\chi(n, \gamma)+\left(\tilde{\delta}(n, \gamma)-2 \chi(n, \gamma) \chi^{\prime}(n, \gamma)\right) \frac{\tilde{\alpha}_{s}\left(q^{2}\right) N_{c}}{4 \pi}\right]
$$

where

$$
\chi^{\prime}(n, \gamma) \equiv \frac{d}{d \gamma} \chi(n, \gamma)=-\Psi^{\prime}\left(\gamma+\frac{n}{2}\right)+\Psi^{\prime}\left(1-\gamma+\frac{n}{2}\right)
$$

Considering the limit $\gamma \rightarrow 0$ we obtain for $n=0$

$$
\begin{aligned}
& \chi(0, \gamma)=\frac{1}{\gamma}+O\left(\gamma^{2}\right), \quad \delta(0, \gamma)-2 \chi(0, \gamma) \chi^{\prime}(0, \gamma)=\frac{A}{\gamma^{2}}+\frac{B}{\gamma}+C+O\left(\gamma^{2}\right), \\
& \tilde{\delta}(0, \gamma)-2 \chi(0, \gamma) \chi^{\prime}(0, \gamma)=\frac{A}{\gamma^{2}}+\frac{\tilde{B}}{\gamma}+C+O\left(\gamma^{2}\right),
\end{aligned}
$$

where

$$
\begin{aligned}
A & =-\left(\frac{11}{3}+\frac{2}{3} \frac{n_{f}}{N_{c}^{3}}\right), B=-\frac{n_{f}}{9 N_{c}^{3}}\left(10 N_{c}^{2}+13\right), \tilde{B}=-\left(\frac{67}{9}-2 \zeta(2)+\frac{13}{9} \frac{n_{f}}{N_{c}^{3}}\right), \\
C & =-\left(\frac{395}{27}-2 \zeta(3)-\frac{11}{3} \zeta(2)+\frac{2 n_{f}}{27 N_{c}^{3}}(71-18 \zeta(2))\right),
\end{aligned}
$$

Following to ref. [7] and using eqs.(34) and (35), one can obtain the expression for anomalous dimensions of twist-2 operators $\gamma$ at $\omega \rightarrow 0$ (i.e. near $j=1$ ) 
in $\overline{M S}$-scheme (coinciding with [7])

$$
\begin{aligned}
\gamma & =\frac{\alpha_{s}\left(q^{2}\right) N_{c}}{\pi}\left[\frac{1}{\omega}+\frac{A}{4}+O(\omega)\right]+\frac{\alpha_{s}^{2}\left(q^{2}\right) N_{c}^{2}}{4 \pi^{2}}\left[\frac{B}{\omega}+O(1)\right] \\
& +\frac{\alpha_{s}^{3}\left(q^{2}\right) N_{c}^{3}}{4 \pi^{3}}\left[\frac{C}{\omega^{2}}+O\left(\omega^{-1}\right)\right]
\end{aligned}
$$

and in GB-scheme

$$
\begin{aligned}
\gamma & =\frac{\alpha_{s}\left(q^{2}\right) N_{c}}{\pi}\left[\frac{1}{\omega}+\frac{A}{4}+O(\omega)\right]+\frac{\alpha_{s}^{2}\left(q^{2}\right) N_{c}^{2}}{4 \pi^{2}}\left[\frac{\tilde{B}}{\omega}+O(1)\right] \\
& +\frac{\alpha_{s}^{3}\left(q^{2}\right) N_{c}^{3}}{4 \pi^{3}}\left[\frac{C}{\omega^{2}}+O\left(\omega^{-1}\right)\right]
\end{aligned}
$$

Note, that the calculation of terms $\sim \alpha_{s}^{3}$ for anomalous dimensions of the twist-2 operators at arbitrary values of $\omega$ will be performed in the near future (see [31] and references therein).

For the case $n \geq 2$ from eq.(33) one can find also the anomalous dimensions for the local operators of the twist $t>2$.

\subsection{The asymptotics of cross-sections at $s \rightarrow \infty$}

As an example we consider the cross-sections for the inclusive production of two pairs of charged particles with mass $m$ in the $\gamma \gamma$ collision (see [2]):

$$
\sigma(s)=\alpha_{e m}^{2} \alpha_{s}^{2} \frac{1}{m^{2}} \frac{32}{81}\left(\sigma_{0}(s)+\left(\cos ^{2} \vartheta-\frac{1}{2}\right) \sigma_{2}(s)\right),
$$

where $\alpha_{e m}$ is electromagnetic coupling constant and $\sigma_{2}(s)$ and $\sigma_{0}(s)$ are the coefficients for angular dependent and independent contributions, respectively.

The asymptotic behaviour of the cross-sections $\sigma_{k}(s)(k=0,2)$ at $s \rightarrow \infty$ corresponds (for the fixed coupling constant) to the unmoving singularities of $f_{\omega}(t)$ of the type $(\omega-$ $\left.\omega_{k}\right)^{1 / 2}$, where

$$
\begin{gathered}
\omega_{k}=\frac{\alpha_{s} N_{c}}{\pi}\left[\chi\left(k, \frac{1}{2}\right)+\frac{\alpha_{s} N_{c}}{4 \pi} \delta\left(k, \frac{1}{2}\right)\right] \equiv \frac{\alpha_{s} N_{c}}{\pi} \chi\left(k, \frac{1}{2}\right)\left[1-\frac{\alpha_{s} N_{c}}{4 \pi} c\left(k, \frac{1}{2}\right)\right], \\
\sigma_{0}(s)=\frac{9 \pi^{5 / 2}}{32 \sqrt{7 \zeta(3)}} \frac{s^{\omega_{0}}}{\left(\ln \left(\frac{s}{s_{0}}\right)\right)^{1 / 2}} \cdot\left(1+O\left(\alpha_{s}\right)\right), \\
\sigma_{2}(s)=\frac{\pi^{5 / 2}}{9 \cdot 32 \sqrt{7 \zeta(3)-8}} \frac{s^{\omega_{2}}}{\left(\ln \left(\frac{s}{s_{0}}\right)\right)^{1 / 2}} \cdot\left(1+O\left(\alpha_{s}\right)\right) .
\end{gathered}
$$

Here the symbol $O\left(\alpha_{s}\right)$ denotes unknown $\alpha_{s}$-corrections to the formfactors. Note, that the running of the QCD coupling constant leads to the substitution of the unmoving cut by an infinite set of the Regge poles [12]. 
Using our results $(26),(27)$, we obtain the following values for $\chi\left(k, \frac{1}{2}\right)$ and $c\left(k, \frac{1}{2}\right)$ $(k=0,2)$ :

$$
\begin{aligned}
\chi\left(0, \frac{1}{2}\right) & =4 \ln 2, \quad \chi\left(2, \frac{1}{2}\right)=4(\ln 2-1) \\
c\left(0, \frac{1}{2}\right) & =2\left(\frac{11}{3}-\frac{2}{3} \frac{n_{f}}{N_{c}}\right) \ln 2-\frac{67}{9}+2 \zeta(2)+\frac{10}{9} \frac{n_{f}}{N_{c}}+\frac{1}{4 \ln 2}\left[22 \zeta(3)-64 \operatorname{Ls}_{3}\left(\frac{\pi}{2}\right)\right. \\
& \left.-\frac{1}{16}\left(271-33 \frac{n_{f}}{N_{c}^{3}}\right) \pi \zeta(2)\right]=25.8388+0.1869 \frac{n_{f}}{N_{c}}+3.8442 \frac{n_{f}}{N_{c}^{3}} \\
c\left(2, \frac{1}{2}\right) & =2\left(\frac{11}{3}-\frac{2}{3} \frac{n_{f}}{N_{c}}\right)(\ln 2-1)-\frac{67}{9}+2 \zeta(2)+\frac{10}{9} \frac{n_{f}}{N_{c}} \\
& +\frac{1}{4(\ln 2-1)}\left[22 \zeta(3)+64 \operatorname{Ls}_{3}\left(\frac{\pi}{2}\right)+\frac{1}{32}\left(893-3 \frac{n_{f}}{N_{c}^{3}}\right) \pi \zeta(2)-64 \ln 2\right] \\
& =-3.2652+1.5203 \frac{n_{f}}{N_{c}}+0.3947 \frac{n_{f}}{N_{c}^{3}}
\end{aligned}
$$

where (see [32] and Appendix A)

$$
\operatorname{Ls}_{3}(x)=-\int_{0}^{x} \ln ^{2}\left|2 \sin \left(\frac{y}{2}\right)\right| d y .
$$

Note, that the function $\operatorname{Ls}_{3}(x)$ appears also in calculations of some massive diagrams (see, for example, the recent articles [33] and references therein).

The LO results (33) coincide with ones obtained in [2]. The value of $c(0,1 / 2)$ equals to $c(1 / 2)$ from [7] (see Appendix A).

As it was discussed in [7], the NLO correction $c(0,1 / 2)$ is large and leads to a quite strong reduction of the value of the Pomeron intercept (see recent analyses [9, 11] of various effective resummations of the large NLO terms). Contrary to $c(0,1 / 2)$, the correction $c(2,1 / 2)$ is very small and does not change the small LO value [2] of the angle-dependent contribution.

In the GB-scheme we have analogously for $k=0,2$ (cf. (31))

$$
\omega_{k}=\frac{\tilde{\alpha}_{s} N_{c}}{\pi}\left[\chi\left(k, \frac{1}{2}\right)+\frac{\tilde{\alpha}_{s} N_{c}}{4 \pi} \tilde{\delta}\left(k, \frac{1}{2}\right)\right] \equiv \frac{\tilde{\alpha}_{s} N_{c}}{\pi} \chi\left(k, \frac{1}{2}\right)\left[1-\frac{\tilde{\alpha}_{s} N_{c}}{4 \pi} \tilde{c}\left(k, \frac{1}{2}\right)\right],
$$

where

$$
\tilde{c}\left(k, \frac{1}{2}\right)=c\left(k, \frac{1}{2}\right)+\frac{67}{9}-2 \zeta(2)-\frac{10}{9} \frac{n_{f}}{N_{c}} .
$$

Note, that in the case of the non-symmetric choice of the scale $s_{0}=q^{2}$ in r.h.s. of eq.(15), the values of the corrections $c(0,1 / 2)$ and $c(2,1 / 2)$ are not changed because $\chi^{\prime}(k, 1 / 2)=0$. Thus, the asymptotics (41) and (42) can be applied directly to the deepinelastic scattering process at small values of Bjorken variable $x$, where $x \approx Q^{2} / s$.

\section{$3 \quad$ NLO corrections to BFKL kernel in supersymmet- ric field theories}




\subsection{Gluino and scalar particle pair production}

As it was shown above, the eigenvalue of the BFKL kernel in the next-to-leading approximation for QCD contains non-analytic contributions proportional to $\delta_{n}^{0}$ and $\delta_{n}^{2}$, which in particular leads to a violation of the holomorphic separability. It is natural to investigate the BFKL equation in supersymmetric gauge theories where there could be significant simplifications. In the case of the $N=1$ supersymmetric Yang-Mills theory we should consider an additional contribution from a gluino loop. It can be obtained from the QCD result for the next-to-leading kernel by a modification of the terms originated from the quark loop contributions proportional to $n_{f}$. Such terms in $\delta(n, \gamma)$ contain the factors $n_{f} / N_{c}$ or $n_{f} / N_{c}^{3}$. The terms $\sim n_{f} / N_{c}$ are related to the quark contribution in the running coupling constant and will be considered below.

We discuss here only the terms in $\delta(n, \gamma)$ proportional to $n_{f} / N_{c}^{3}$. Their contribution to $\omega$ depends on $\gamma=1 / 2+i \nu$ and can be written as follows

$$
\omega_{n_{f} / N_{c}^{3}}=-\frac{n_{f}}{4 N_{c}}\left(\frac{\alpha_{s}}{\alpha}\right)^{2} \omega_{Q E D},
$$

where according to eq. (B1) from Appendix B

$$
\omega_{Q E D}=\frac{\alpha^{2} \cos (\pi \gamma)}{\sin ^{2}(\pi \gamma)(1-2 \gamma)}\left(\frac{(2+3 \gamma(1-\gamma)) \delta_{n}^{0}}{(3-2 \gamma)(1+2 \gamma)}-\frac{\gamma(1-\gamma) \delta_{|n|}^{2}}{2(3-2 \gamma)(1+2 \gamma)}\right)
$$

coincides with the position of the Pomeranchuck singularity of the $t$-channel partial wave $f_{\omega}(t)$ in QED (see [34]). To give an physical interpretation of this result one should take into account, that in the $\omega$-plane the BFKL pomeron is the Mandelstam cut originated from an exchange of two reggeized gluons. It means, that the amplitude for the transition of two reggeized gluons into two others in the $t$-channel through the fermion loop should contain the third spectral function $\rho(s, u)$. Therefore after the substitution $\alpha \rightarrow \alpha_{s}$ it should coincide with the amplitude for the virtual photon-photon scattering in QED up to the colour factor

$$
-\frac{n_{f}}{4 N_{c}}=n_{f} \frac{1}{N_{c}^{2}-1} \operatorname{tr}\left(t^{a} t^{b} t^{a} t^{b}\right),\left[t^{a}, t^{b}\right]=i f^{a b c} t^{c}
$$

for the corresponding non-planar loop diagram.

Taking into account, that gluino is a Mayorana particle contrary to the charged electron and that its field belongs to the adjoint representation $\left(T^{a}\right)^{b c}=-i f^{a b c}$ of the gauge group, we obtain for the gluino contribution in the $N$-extended supersymmetric gauge theory the following result

$$
\omega_{\text {gluino }}=\frac{N}{2} \frac{N_{c}^{2}}{2}\left(\frac{\alpha_{s}}{\alpha}\right)^{2} \omega_{Q E D} .
$$

Providing that $N>1$, there is also a contribution of scalar super-partners of the gluon:

$$
\omega_{\text {scalar }}=\frac{n_{s}(N)}{2} \frac{N_{c}^{2}}{2}\left(\frac{\alpha_{s}}{\alpha}\right)^{2} \omega_{S E D},
$$


where $n_{s}(N)$ is the total number of scalar particles and $\omega_{S E D}$ is the position of the Pomeranchuck singularity in the scalar electrodynamics (see eq.(B2) from Appendix B and ref. [34]):

$$
\omega_{S E D}=\frac{\alpha^{2} \cos (\pi \gamma)}{\sin ^{2}(\pi \gamma)(1-2 \gamma)}\left(\frac{(1+\gamma(1-\gamma)) \delta_{n}^{0}}{2(3-2 \gamma)(1+2 \gamma)}+\frac{\gamma(1-\gamma) \delta_{|n|}^{2}}{4(3-2 \gamma)(1+2 \gamma)}\right) .
$$

For the number of scalar particles in the $N$-extended supersymmetric theories we have in the case of $N=2$ and $N=4$

$$
n_{s}(2)=2, n_{s}(4)=6
$$

taking into account, that for $N=4$ the gluons and gluinos with both helicities $\lambda= \pm s$ enter in one super-multiplet with the scalar particles. In particular, the total contribution to $\omega$ from super-partners of the gluon for $N=4$ is

$$
\begin{gathered}
\Delta \omega_{s p}=N_{c}^{2}\left(\frac{\alpha_{s}}{\alpha}\right)^{2}\left(\omega_{Q E D}+\frac{3}{2} \omega_{S E D}\right)= \\
\frac{N_{c}^{2} \alpha_{s}^{2} \cos (\pi \gamma)}{\sin ^{2}(\pi \gamma)(1-2 \gamma)}\left(\frac{(11+15 \gamma(1-\gamma)) \delta_{n}^{0}}{4(3-2 \gamma)(1+2 \gamma)}-\frac{\gamma(1-\gamma) \delta_{|n|}^{2}}{8(3-2 \gamma)(1+2 \gamma)}\right) .
\end{gathered}
$$

It is remarkable, that this term cancels the corresponding gluon contribution non-analytic in $n$ (see (23) and (27)):

$$
\Delta \omega_{g}=-\frac{N_{c}^{2} \alpha_{s}^{2} \cos (\pi \gamma)}{4 \sin ^{2}(\pi \gamma)(1-2 \gamma)}\left(\left(3+\frac{(2+3 \gamma(1-\gamma))}{(3-2 \gamma)(1+2 \gamma)}\right) \delta_{n}^{0}-\frac{\gamma(1-\gamma)}{2(3-2 \gamma)(1+2 \gamma)} \delta_{|n|}^{2}\right) .
$$

\subsection{Scalar particle contribution to the gluon Regge trajectory}

Let us consider the contribution of scalar particles to the gluon Regge trajectory in the two-loop approximation. We use the results of ref. [22], where the corresponding quark correction was calculated, which gives a possibility to find easily the gluino correction using the substitution $n_{f} \rightarrow N N_{c}$. The quark contribution can be presented in the form

$$
\begin{aligned}
\omega_{n_{f} / N_{c}}^{(2)}(q) & =-\frac{2 n_{f}}{3 N_{c}} \cdot \frac{\alpha^{2} q^{2}}{2} \int \frac{d^{D-2} \tilde{q}}{\tilde{q}^{2}(\tilde{q}-q)^{2}}\left[2 \Pi_{q}(\tilde{q})-\Pi_{q}(q)\right], \\
\Pi_{q}(q) & =-\frac{2(1+\varepsilon)}{(3+2 \varepsilon)(1+2 \varepsilon) \varepsilon} \frac{\Gamma^{2}(1+\varepsilon)}{\Gamma(1+2 \varepsilon)} \frac{\Gamma(1-\varepsilon)}{\left(q^{2}\right)^{-\varepsilon}},
\end{aligned}
$$

where $\alpha \Pi_{q}(q)\left(g^{\mu \nu} q^{2}-q^{\mu} q^{\nu}\right)$ is one-loop quark self-energy correction to the polarization tensor, and $\alpha$ the is bare QCD constant

$$
\alpha=\frac{g^{2} N_{c} \Gamma(1-\varepsilon)}{(4 \pi)^{2+\varepsilon}}
$$

with well-known relation between $g_{\mu}^{2}$ and $g^{2}$ in $\overline{M S}$ scheme:

$$
g^{2}=g_{\mu}^{2} \mu^{-2 \varepsilon}\left[1+\frac{1}{\varepsilon}\left(\frac{11}{3}-\frac{2}{3} \frac{n_{f}}{N_{c}}\right) g_{\mu}^{2}\right]
$$


where the last term in the r.h.s. of Eq.(51) is proportional to the leading order term $\beta_{0}$ (30) of QCD $\beta$-function.

The scalar particle correction can be presented in the similar form

$$
\begin{aligned}
\omega_{s c}^{(2)}(q) & =-\frac{2 n_{s}(N)}{3 N_{c}} \cdot \frac{\bar{a}^{2} q^{2}}{2} \int \frac{d^{D-2} \tilde{q}}{\tilde{q}^{2}(\tilde{q}-q)^{2}}\left[2 \Pi_{s c}(\tilde{q})-\Pi_{s c}(q)\right] \\
\Pi_{s c}(q) & =-\frac{1}{2(3+2 \varepsilon)(1+2 \varepsilon) \varepsilon} \frac{\Gamma^{2}(1+\varepsilon)}{\Gamma(1+2 \varepsilon)} \frac{\Gamma(1-\varepsilon)}{\left(q^{2}\right)^{-\varepsilon}},
\end{aligned}
$$

where $\bar{a} \Pi_{s c}(q)\left(g^{\mu \nu} q^{2}-q^{\mu} q^{\nu}\right)$ is one-loop self-energy correction to the polarization tensor from scalar particles and $\bar{a}$ is the bare constant of $N=4$ supersymmetric theory

$$
\bar{a}=\frac{\bar{g}^{2} N_{c}}{(4 \pi)^{2}}
$$

As it is well known, the coupling constant of the $N=4$ supersymmetric theory does not run, i.e. its $\beta$-function is zero, and, hence, $\bar{a}=\bar{a}_{\mu}$.

\subsection{The results of calculations}

Putting all things together, in the case of $N=4$ supersymmetric theory we have the following representation for the Regge trajectory

$$
\begin{gathered}
\omega^{\text {susy }}(q)=-\bar{a}_{\mu}\left(\frac{2}{\varepsilon}+2 \ln \left(\frac{q^{2}}{\mu^{2}}\right)\right) \\
-\bar{a}_{\mu}^{2}\left[\left(\frac{1}{3}-2 \zeta(2)\right)\left(\frac{1}{\varepsilon}+2 \ln \left(\frac{q^{2}}{\mu^{2}}\right)\right)-\frac{8}{9}+2 \zeta(3)\right],
\end{gathered}
$$

Using (53), by analogy with the results of [0] for QCD we can easily find the corresponding kernel $K_{r}^{\text {susy }}\left(q_{1}, q_{2}\right)$ for the $N=4$ SUSY

$$
\begin{gathered}
K_{r}^{\text {susy }}\left(q_{1}, q_{2}\right)=\frac{4 \bar{a}_{\mu} \mu^{-2 \varepsilon}}{\pi^{1+\varepsilon} \Gamma(1-\varepsilon)}\left\{\frac{1}{\left(q_{1}-q_{2}\right)^{2}}(1\right. \\
\left.+\bar{a}_{\mu}\left[\left(\frac{\left(q_{1}-q_{2}\right)^{2}}{\mu^{2}}\right) \varepsilon\left(\frac{1}{3}-2 \zeta(2)+\varepsilon\left(-\frac{8}{9}+14 \zeta(3)\right)\right)-\ln ^{2}\left(\frac{q_{1}^{2}}{q_{2}^{2}}\right)\right]\right) \\
+\bar{a}_{\mu}\left[\frac{\left(q_{1}^{2}-q_{2}^{2}\right)}{\left(q_{1}-q_{2}\right)^{2}\left(q_{1}+q_{2}\right)^{2}}\left(\ln \left(\frac{q_{1}^{2}}{q_{2}^{2}}\right) \ln \left(\frac{q_{1}^{2} q_{2}^{2}\left(q_{1}-q_{2}\right)^{4}}{\left(q_{1}^{2}+q_{2}^{2}\right)^{4}}\right)-2 \operatorname{Li}_{2}\left(-\frac{q_{1}^{2}}{q_{2}^{2}}\right)+2 \operatorname{Li}_{2}\left(\frac{q_{1}^{2}}{q_{2}^{2}}\right)\right)\right. \\
\left.\left.-\left(1-\frac{\left(q_{1}^{2}-q_{2}^{2}\right)}{\left(q_{1}-q_{2}\right)^{2}\left(q_{1}+q_{2}\right)^{2}}\right)\left(\int_{0}^{1}-\int_{1}^{\infty}\right) \frac{d x}{\left(q_{2}-x q_{1}\right)^{2}} \ln \left(\frac{x q_{1}^{2}}{q_{2}^{2}}\right)\right]\right\},
\end{gathered}
$$

The set of eigenvalues for eigenfunctions (22) of the homogeneous BFKL equation in the framework of $N=4$ supersymmetric theory

$$
\omega^{\text {susy }}=4 \bar{a}\left[\chi(n, \gamma)+\delta^{\text {susy }}(n, \gamma) \bar{a}\right]
$$


can be found analogously to the previous section in the following form

$$
\begin{aligned}
\delta^{\text {susy }}(n, \gamma) & =-\left[2 \Phi(n, \gamma)+2 \Phi(n, 1-\gamma)-\left(\frac{1}{3}-2 \zeta(2)\right) \chi(n, \gamma)\right. \\
& \left.-6 \zeta(3)-\Psi^{\prime \prime}\left(\gamma+\frac{n}{2}\right)-\Psi^{\prime \prime}\left(1-\gamma+\frac{n}{2}\right)\right],
\end{aligned}
$$

where the function $\Phi(n, \gamma)$ was defined in eq.(28).

Note, that the term

$$
\left(\frac{1}{3}-2 \zeta(2)\right) \chi(n, \gamma)
$$

appears as a result of the use of the $\overline{M S}$-scheme in intermediate calculations (see (29)). This term can be eliminated by a redefinition of the coupling constant

$$
\bar{a} \rightarrow \tilde{a}=\bar{a}+\left(\frac{1}{3}-2 \zeta(2)\right) \bar{a}^{2}
$$

which is equivalent to the use of the BG-scheme (see (30)). For the new coupling constant $\tilde{a}$ above results can be written in the following form

$$
\omega^{\text {susy }}=4 \tilde{a}\left[\chi(n, \gamma)+\tilde{\delta}^{\text {susy }}(n, \gamma) \tilde{a}\right]
$$

where

$$
\tilde{\delta}^{\text {susy }}(n, \gamma)=-\left[2 \Phi(n, \gamma)+2 \Phi(n, 1-\gamma)-6 \zeta(3)-\Psi^{\prime \prime}\left(\gamma+\frac{n}{2}\right)-\Psi^{\prime \prime}\left(1-\gamma+\frac{n}{2}\right)\right]
$$

Analogously to the previous section and ref. [7] we can obtain the eigenvalues of the kernel in the case of non-symmetric choice of the energy-normalization $s_{0}$ in eq.(15). For the scale $s_{0}=q^{2}$ we have

in $\overline{M S}$-scheme

$$
\omega^{\text {susy }}=4 \bar{a}\left[\chi(n, \gamma)+\left(\delta^{\text {susy }}(n, \gamma)-2 \chi(n, \gamma) \chi^{\prime}(n, \gamma)\right) \bar{a}\right]
$$

and in GB-scheme

$$
\omega^{\text {susy }}=4 \tilde{a}\left[\chi(n, \gamma)+\left(\tilde{\delta}^{\text {susy }}(n, \gamma)-2 \chi(n, \gamma) \chi^{\prime}(n, \gamma)\right) \tilde{a}\right]
$$

The anomalous dimensions $\gamma^{\text {susy }}$ of the twist- 2 operators at $\omega \rightarrow 0$ (and $n=0$ ) are in $\overline{M S}$-scheme

$$
\gamma^{\text {susy }}=4 \bar{a}\left[\left(\frac{1}{\omega}+O(\omega)\right)+\bar{a}\left(\frac{B^{\text {susy }}}{\omega}+O(1)\right)+\bar{a}^{2}\left(\frac{C^{\text {susy }}}{\omega^{2}}+O\left(\omega^{-1}\right)\right)\right]
$$

and in GB-scheme

$$
\gamma^{\text {susy }}=4 \tilde{a}\left[\left(\frac{1}{\omega}+O(\omega)\right)+\tilde{a}\left(\frac{\tilde{B}^{\text {susy }}}{\omega}+O(1)\right)+\tilde{a}^{2}\left(\frac{C^{\text {susy }}}{\omega^{2}}+O\left(\omega^{-1}\right)\right)\right],
$$


where

$$
B^{\text {susy }}=\frac{1}{3}, \quad \tilde{B}^{\text {susy }}=2 \zeta(2), \quad \text { and } \quad C^{\text {susy }}=2 \zeta(3) .
$$

For the case $n \geq 2$ one can calculate also the anomalous dimensions of the corresponding local operators. Because in the case $N=4$ SUSY the result is analytic in $|n|$, one can continue these anomalous dimensions to the negative values of $|n|$. It gives a possibility to find the singular contributions of the anomalous dimensions of the twist-2 operators not only at $j=1$ but also at other integer points $j=0,-1,-2 \ldots$ In particular, in the Born approximation for the anomalous dimension of the operator corresponding to the sum of distributions of gluons, gluino and scalar particles we obtain $\gamma=4 \bar{a}(\Psi(1)-\Psi(j-1))$ which coincides with the result of the direct calculations (see [20]). Thus, in the case $N=4$ the BFKL equation presumably contains the information sufficient for restoring the kernel of the DGLAP equation.

\section{Conclusion}

In this paper we calculated next-to-leading corrections to eigenvalues of the BFKL kernel in QCD for arbitrary conformal spins $n$ (see (27)). Now with the use of eqs. (39), (42) one can find the spin-dependent contribution to the total cross-section for the $\gamma^{*} \gamma^{*}$ annihilation into hadrons at high energies. We calculated also next-to-leading corrections to the BFKL equation in the supersymmetric field theories and next-to-leading corrections to anomalous dimensions of twist-2 operators (see (56), (59)). In a particular case of the extended $N=4$ SUSY the result of our calculations is significantly simplified (see eqs. (56) and (57)). The absence of the coupling constant renormalization in this model leads presumable to the Möbius invariance of the BFKL equation in higher orders of the perturbation theory. This invariance can allow one to guess the next-to-leading corrections to the BFKL kernel in QCD for a general case $t \neq 0$, which is very important for the theory of the processes at small $x$. The remarkable cancellation of non-analytic contributions proportional to $\delta_{n}^{0}$ and $\delta_{n}^{2}$ at $N=4$ SUSY is a possible manifestation of the integrability of the reggeon dynamics in the Maldacena model [21] corresponding to the limit $N_{c} \rightarrow$ $\infty$. Note, that in this model the eigenvalues of the LLA pair kernels in the evolution equations for the matrix elements of the quasi-partonic operators are proportional to $\psi(j-1)-\psi(1)$ [20], which means, that the corresponding Hamiltonian coincides with the local Hamiltonian for the integrable Heisenberg spin model. The residues of these eigenvalues in the points $j=-k$ can be obtained from the BFKL equation by the analytic continuation of the anomalous dimensions to negative integer values of the conformal spin $|n|$. Therefore the DGLAP equation is not independent from the BFKL equation in $N=4$ SUSY and their integrability properties at $N_{c} \rightarrow \infty$ are related. We shall return to these problems in our future publications.

\section{Acknowledgments}


A. Kotikov thanks the Alexander von Humboldt Foundation for its support. L. Lipatov thanks the Hamburg University for its hospitality during the period of time when this work was done. He is supported by the INTAS grant 97-31696 and by the Deutsche Forschungsgemeinschaft.

We are indebted to J. Bartels, V. Fadin, V. Kim, R. Kirschner, R. Peschansky and participants of the PNPI Winter School and of the Gribov-70 Workshop for helpful discussions.

\section{A Appendix}

Here we demonstrate how the function $\operatorname{Ls}_{3}(x)$ appears in eqs. (41) and (42).

The direct evaluation of the values of $c(0,1 / 2)$ and $c(2,1 / 2)$ leads to

$$
\begin{aligned}
c\left(0, \frac{1}{2}\right) & =2\left(\frac{11}{3}-\frac{2}{3} \frac{n_{f}}{N_{c}}\right) \ln 2-\frac{67}{9}+2 \zeta(2)+\frac{10}{9} \frac{n_{f}}{N_{c}}+\frac{1}{4 \ln 2}[22 \zeta(3) \\
& \left.+16 \int_{0}^{1} \frac{d t}{t} \arctan (\sqrt{t}) \ln \left(\frac{1}{1-t}\right)+\frac{1}{16}\left(81+33 \frac{n_{f}}{N_{c}^{3}}\right) \pi \zeta(2)\right] \\
c\left(2, \frac{1}{2}\right) & =2\left(\frac{11}{3}-\frac{2}{3} \frac{n_{f}}{N_{c}}\right)(\ln 2-1)-\frac{67}{9}+2 \zeta(2)+\frac{10}{9} \frac{n_{f}}{N_{c}}+\frac{1}{4(\ln 2-1)}[22 \zeta(3) \\
& \left.-16 \int_{0}^{1} \frac{d t}{t} \arctan (\sqrt{t}) \ln \left(\frac{1}{1-t}\right)+\frac{1}{32}\left(189-3 \frac{n_{f}}{N_{c}^{3}}\right) \pi \zeta(2)-64 \ln 2\right]
\end{aligned}
$$

Note that (A.1) coincides exactly with eq.(16) of [7].

To obtain eqs. (41) and (42) we should evaluate the integral

$$
\int_{0}^{1} \frac{d t}{t} \arctan (\sqrt{t}) \ln \left(\frac{1}{1-t}\right)
$$

Using the following substitutions

$$
t=p^{2}, \quad \arctan (x+i y)=\frac{i}{2} \ln \frac{1+y-i x}{1-y+i x}+2 \pi k \quad\left(i=e^{i \pi / 2}\right),
$$

we obtain for it the following representation (for $k=0$ )

$$
\begin{aligned}
\int_{0}^{1} \frac{d t}{t} \arctan (\sqrt{t}) \ln \left(\frac{1}{1-t}\right) & =-i \int_{0}^{1} \frac{d p}{p}(\ln (1-p)+\ln (1+p)) \\
\cdot(\ln (1-i p)-\ln (1+i p)) & =i\left[\mathrm{~F}_{1}(i)+\mathrm{F}_{2}(i)-\mathrm{F}_{1}(-i)-\mathrm{F}_{2}(-i)\right],
\end{aligned}
$$

where [35]

$$
\begin{aligned}
\mathrm{F}_{1}(a) & \equiv \int_{0}^{1} \frac{d p}{p} \ln (1-p) \ln (1+a p)=\operatorname{Li}_{3}(-a)+\mathrm{S}_{1,2}(-a) \quad \text { and } \\
\mathrm{F}_{2}(a) & \equiv \int_{0}^{1} \frac{d p}{p} \ln (1+p) \ln (1+a p)=\mathrm{S}_{1,2}\left(\frac{1-a}{1+a}\right)-\mathrm{S}_{1,2}\left(\frac{a-1}{1+a}\right)+\mathrm{S}_{1,2}(-a) \\
& +\operatorname{Li}_{3}(a)-\frac{7}{8} \zeta(3)+\frac{1}{2} \ln ^{2} a \ln \left(\frac{1-a}{1+a}\right)-\ln a\left[\zeta(2)-\operatorname{Li}_{2}\left(\frac{1-a}{1+a}\right)\right]
\end{aligned}
$$


Here $\mathrm{S}_{n, p}(x)$ are the Nielsen generalized polylogarithms (see [36, 35]):

$$
\mathrm{S}_{n, p}(x)=\frac{(-1)^{n+p-1}}{(n-1) ! p !} \int_{0}^{1} \frac{d y}{y} \ln ^{n-1} y \ln (1-x y)
$$

and the polylogarithms $\operatorname{Li}_{n}(x)=\mathrm{S}_{n-1,1}(x)$.

With the use of (A.4) we have

$$
\begin{aligned}
& \mathrm{F}_{1}( \pm i)=\mathrm{Li}_{3}(\mp i)+\mathrm{S}_{1,2}(\mp i) \\
& \mathrm{F}_{2}( \pm i)=2 \mathrm{~S}_{1,2}(\mp i)-\mathrm{S}_{1,2}( \pm i)+\mathrm{Li}_{3}( \pm i)-\frac{7}{8} \zeta(3) \pm \frac{\pi}{2} i\left[\operatorname{Li}_{2}(\mp i)+\frac{1}{2} \zeta(2)\right]
\end{aligned}
$$

Using the integral representation (A.5), we have for the Euler dilogarithm $\operatorname{Li}_{2}(i)$ :

$$
\operatorname{Li}_{2}(1)-\operatorname{Li}_{2}(i)=\int_{1}^{i} \frac{d p}{p} \ln (1-p) .
$$

If we replace $s$ by $s=e^{i \theta}$ corresponding to

$$
1-s=1-e^{i \theta}=2 \sin \left(\frac{\theta}{2}\right) e^{i(\theta-\pi) / 2}
$$

we obtain

$$
\mathrm{Li}_{2}(1)-\mathrm{Li}_{2}(i)=\frac{9}{8} \zeta(2)-i \mathrm{G}
$$

where

$$
\mathrm{G} \equiv-\int_{0}^{1} d x \frac{\ln x}{1+x^{2}}=\mathrm{Cl}_{2}(\pi / 2)
$$

Here according to ref. 32

$$
\mathrm{Cl}_{2}(x)=\mathrm{Ls}_{2}(x) \quad \text { and } \quad \operatorname{Ls}_{n}(x)=-\int_{0}^{x} \ln ^{n-1}\left|2 \sin \left(\frac{y}{2}\right)\right| d y .
$$

Thus,

$$
\mathrm{Li}_{2}( \pm i)=-\frac{1}{8} \zeta(2) \pm i \mathrm{G}
$$

Repeating above calculations, one can derive the relations

$$
\begin{aligned}
\mathrm{Li}_{3}( \pm i) & =-\frac{3}{32} \zeta(3) \pm \frac{3}{16} i \pi \zeta(2) \\
\mathrm{S}_{1,2}( \pm i) & =\frac{29}{64} \zeta(3)-\frac{\pi}{4} \mathrm{G} \mp \frac{i}{2}\left[\operatorname{Ls}_{3}\left(\frac{\pi}{2}\right)+\frac{7}{16} \pi \zeta(2)\right]
\end{aligned}
$$

The obtained results (A.7)-(A.9) are in agreement with ref. 32.

Putting (A.7)-(A.9) to (A.6) and (A.3), we have

$$
\int_{0}^{1} \frac{d t}{t} \arctan (\sqrt{t}) \ln \left(\frac{1}{1-t}\right)=-4 \operatorname{Ls}_{3}\left(\frac{\pi}{2}\right)-\frac{11}{8} \pi \zeta(2)
$$

and, thus, eqs.(A.1) and (A.2) coincide with Eqs.(41) and (42), respectively. 


\section{B Appendix}

Although the Pomeranchuck singularity in QED was investigated many years ago (see [34]), here we review the obtained results and generalize them to the case of the charged scalar particle production.

To begin with, let us consider the electron-electron scattering in the Regge kinematics $2 p_{A} p_{B}=s \gg q^{2}$, where $p_{A}$ and $p_{B}$ are momenta of the colliding electrons. In the Born approximation the corresponding amplitude equals

$$
A_{e e \rightarrow e e}=j_{\mu}\left(p_{A}\right) \frac{\delta^{\mu \nu}}{q^{2}} j_{\nu}\left(p_{B}\right)=j_{\mu}\left(p_{A}\right) p_{B}^{\mu} \frac{2 / s}{q^{2}} j_{\nu}\left(p_{B}\right) p_{A}^{\nu}=2 e^{2} \frac{s}{q^{2}},
$$

where $j_{\mu}(p)=e \bar{u}\left(p^{\prime}\right) \gamma_{\mu} u(p)$. The elastic cross-section is

$$
\sigma_{e l}=\frac{1}{4} \int \frac{d^{2} q}{(2 \pi)^{2}} \frac{\left|A_{e e \rightarrow e e}\right|^{2}}{s^{2}}=4 \alpha^{2} \int \frac{d^{2} q}{|q|^{4}} .
$$

This cross-section is divergent at small $q$ due to an infinite radius of the Coulomb interaction. We shall ignore this circumstance because in the case of the scattering of non-charged particles $A$ and $B$ the integrand is multiplied by a product of their impact factors $\Phi_{A}(q)$ and $\Phi_{B}(q)$ vanishing at $q=0$

$$
\sigma_{A B}=\int \frac{d^{2} q}{(2 \pi)^{2}|q|^{4}} \Phi_{A}(q) \Phi_{B}(q)
$$

which leads to the cancellation of this divergency (see [34]). Note, that for the electron being a charged particle the impact factor is non-zero for $q=0$ :

$$
\Phi_{e}(q)=4 \pi \alpha
$$

The amplitude for the production of a massless electron-positron pair in the ee scattering is

$$
A_{e e \rightarrow e e e^{+} e}=2 e^{4} \frac{s}{q_{1}^{2} q_{2}^{2}} A_{\gamma * \gamma * \rightarrow e^{+} e}
$$

where the factor $A_{\gamma * \gamma * \rightarrow e^{+}}$describes the transition of two virtual photons into the $e^{+} e^{-}$ pair

$$
A_{\gamma * \gamma * \rightarrow e^{+} e^{-}}=\bar{u}_{e^{-}}\left(k_{1}\right) A_{e} v_{e^{+}}\left(-k_{2}\right), q_{1}-q_{2}=k_{1}+k_{2}
$$

The amplitude $A_{e}$ is given below

$$
A_{e}=\frac{2}{s}\left(\widehat{p_{A}} \frac{\widehat{k_{1}^{\perp}}-\widehat{q_{1}^{\perp}}}{\left|k_{1}-q_{1}\right|^{2}+\left|k_{1}\right|^{2} \frac{\beta_{2}}{\beta_{1}}} \widehat{p_{B}}+\widehat{p_{B}} \frac{-\widehat{k_{2}^{\perp}}+\widehat{q_{1}^{\perp}}}{\left|k_{2}-q_{1}\right|^{2}+\left|k_{2}\right|^{2} \frac{\beta_{1}}{\beta_{2}}} \widehat{p_{A}}\right),
$$

where we introduced the Sudakov variables for momenta $k_{1}$ and $k_{2}$ of the produced electron and positron 


$$
k_{r}=\alpha_{r} p_{B}+\beta_{r} p_{A}+k_{r}^{\perp}, s \alpha_{r} \beta_{r}=-\left(k_{r}^{\perp}\right)^{2}=\left|k_{r}\right|^{2}
$$

and used for the momenta of the virtual photons the following relations

$$
q_{1}=\left(\beta_{1}+\beta_{2}\right) p_{A}+q_{1}^{\perp}, q_{2}=-\left(\alpha_{1}+\alpha_{2}\right) p_{B}+q_{2}^{\perp}
$$

valid at high energies when $2 k_{1} k_{2} \ll s$ (corresponding to small $\alpha_{r}$ and $\beta_{r}$ ). Note, that with the use of the Dirac equation for the produced particles we can rewrite $A_{e}$ in the other form

$$
A_{e} \rightarrow \frac{2}{s\left(\beta_{1}+\beta_{2}\right)}\left(\frac{s \beta_{2} \widehat{q_{1}^{\perp}}+\widehat{q_{1}^{\perp}} \widehat{q_{2}^{\perp}} \widehat{p_{B}}}{\left|k_{1}-q_{1}\right|^{2}+\left|k_{1}\right|^{2} \frac{\beta_{2}}{\beta_{1}}}-\frac{s \beta_{1} \widehat{q_{1}^{\perp}}+\widehat{p_{B}} \widehat{q_{2}^{\perp}} \widehat{q_{1}^{\perp}}}{\left|k_{1}+q_{2}\right|^{2}+\left|k_{1}-q_{1}+q_{2}\right|^{2} \frac{\beta_{1}}{\beta_{2}}}\right)
$$

and verify its vanishing at $q_{1}^{\perp}=0$ or $q_{2}^{\perp}=0$.

Analogously for the production of a pair of charged scalar particles $s$ we have

$$
A_{e e \rightarrow e e s^{+} s^{-}}=2 e^{4} \frac{s}{q_{1}^{2} q_{2}^{2}} A_{s}
$$

where

$$
A_{s}=-2+\frac{2\left|k_{1}\right|^{2}}{\left|k_{1}-q_{1}\right|^{2}+\left|k_{1}\right|^{2} \frac{\beta_{2}}{\beta_{1}}}+\frac{2\left|k_{2}\right|^{2}}{\left|k_{2}-q_{1}\right|^{2}+\left|k_{2}\right|^{2} \frac{\beta_{1}}{\beta_{2}}} .
$$

It can be written in the form

$$
A_{s} \rightarrow \frac{2}{\beta_{1}+\beta_{2}}\left(\frac{\beta_{2}\left(\overrightarrow{q_{1}}, 2 \overrightarrow{k_{1}}-\overrightarrow{q_{1}}\right)}{\left|k_{1}-q_{1}\right|^{2}+\left|k_{1}\right|^{2} \frac{\beta_{2}}{\beta_{1}}}+\frac{\beta_{1}\left(\overrightarrow{q_{1}}, 2 \overrightarrow{k_{2}}-\overrightarrow{q_{1}}\right)}{\left|k_{2}-q_{1}\right|^{2}+\left|k_{2}\right|^{2} \frac{\beta_{1}}{\beta_{2}}}\right)
$$

to make obvious its vanishing at $q_{1}=0$ or at $q_{2}=0$. The total cross-section for the pair production in the collision of the particles $A$ and $B$ after calculating the integrals over $\alpha_{i}$ $(i=1,2)$ with the use of the $\delta$-functions $\delta\left(s \alpha_{i} \beta_{i}-\left|k_{i}\right|^{2}\right)$ can be presented as follows

$$
\sigma_{\text {pair }}=\log \frac{s}{m^{2}} \int \frac{d^{2} q_{1} \Phi_{A}\left(q_{1}\right)}{(2 \pi)^{2}\left|q_{1}\right|^{4}} \frac{d^{2} q_{1} \Phi_{B}\left(q_{2}\right)}{(2 \pi)^{2}\left|q_{2}\right|^{4}} K\left(\overrightarrow{q_{1}}, \overrightarrow{q_{2}}\right) \text {. }
$$

The kernel $K\left(\overrightarrow{q_{1}}, \overrightarrow{q_{2}}\right)$ for electrons $e$ and scalar particles $s$ is correspondingly

$$
K_{e, s}\left(\overrightarrow{q_{1}}, \overrightarrow{q_{2}}\right)=16 \alpha^{2} \int \frac{d^{2} k_{1}}{(2 \pi)^{2}} \int_{0}^{1} d x f_{e, s}\left(\overrightarrow{k_{1}} ; x\right), x=\frac{\beta_{1}}{\beta_{1}+\beta_{2}}
$$

and

$$
f_{e}\left(\overrightarrow{k_{1}} ; x\right)=\frac{\operatorname{tr}\left(\widehat{k_{1}} A_{e} \widehat{k_{2}} A_{e}^{+}\right)}{16 x(1-x)}, \quad f_{s}\left(\overrightarrow{k_{1}} ; x\right)=\frac{\left|A_{s}\right|^{2}}{16 x(1-x)} .
$$

The factor $\log \left(s / m^{2}\right)$ was obtained as a result of the integration over $\beta=\beta_{1}+\beta_{2}$ in the region $m^{2} / s \ll \beta \ll 1$, where the mass scale $m$ equals to an essential value for 
transverse momenta of charged particles inside the colliding particles. We obtain for the $e^{+} e^{-}$production

$$
\begin{aligned}
& f_{e}\left(\overrightarrow{k_{1}} ; x\right)=\frac{\frac{1}{2}\left|k_{1}\right|^{2}\left|k_{1}-q_{1}\right|^{2}}{\left(\left|k_{1}-x q_{1}\right|^{2}+x(1-x)\left|q_{1}\right|^{2}\right)^{2}}+\frac{\frac{1}{2}\left|k_{2}\right|^{2}\left|k_{1}+q_{2}\right|^{2}}{\left(\left|k_{1}+q_{2}-x q_{1}\right|^{2}+x(1-x)\left|q_{2}\right|^{2}\right)^{2}}- \\
& \frac{\left(\overrightarrow{k_{1}}-\overrightarrow{q_{1}}, \overrightarrow{q_{2}}+\overrightarrow{k_{1}}\right)\left(\overrightarrow{k_{2}}, \overrightarrow{k_{1}}\right)-\left(\overrightarrow{k_{1}}-\overrightarrow{q_{1}}, \overrightarrow{k_{2}}\right)\left(\overrightarrow{k_{1}}+\overrightarrow{q_{2}}, \overrightarrow{k_{1}}\right)-\left(\overrightarrow{k_{1}}+\overrightarrow{q_{2}}, \overrightarrow{k_{2}}\right)\left(\overrightarrow{k_{1}}-\overrightarrow{q_{1}}, \overrightarrow{k_{1}}\right)}{\left(\left|k_{1}-x q_{1}\right|^{2}+x(1-x)\left|q_{1}\right|^{2}\right)\left(\left|k_{1}+q_{2}-x q_{1}\right|^{2}+x(1-x)\left|q_{1}\right|^{2}\right)}
\end{aligned}
$$

and for charged scalar pair production

$$
\begin{gathered}
f_{s}\left(\overrightarrow{k_{1}} ; x\right)=\frac{\frac{1}{4} x(1-x)\left(\overrightarrow{q_{1}}, 2 \overrightarrow{k_{1}}-\overrightarrow{q_{1}}\right)^{2}}{\left(\left|k_{1}-x q_{1}\right|^{2}+x(1-x)\left|q_{1}\right|^{2}\right)^{2}}+\frac{\frac{1}{4} x(1-x)\left(\overrightarrow{q_{1}}, 2 \overrightarrow{k_{2}}-\overrightarrow{q_{1}}\right)^{2}}{\left(\left|k_{1}+q_{2}-x q_{1}\right|^{2}+x(1-x)\left|q_{2}\right|^{2}\right)^{2}}+ \\
\frac{\frac{1}{2} x(1-x)\left(\overrightarrow{q_{1}}, 2 \overrightarrow{k_{1}}-\overrightarrow{q_{1}}\right)\left(\overrightarrow{q_{1}}, 2 \overrightarrow{k_{2}}-\overrightarrow{q_{1}}\right)}{\left(\left|k_{1}-x q_{1}\right|^{2}+x(1-x)\left|q_{1}\right|^{2}\right)\left(\left|k_{1}+q_{2}-x q_{1}\right|^{2}+x(1-x)\left|q_{1}\right|^{2}\right)} .
\end{gathered}
$$

By introducing the Feynman parameter $y$ to unify two factors in the denominators of the last contributions to $f_{e}\left(\overrightarrow{k_{1}} ; x\right)$ and $f_{s}\left(\overrightarrow{k_{1}} ; x\right)$ and integrating the kernel over $\overrightarrow{k_{1}}$ one can present it in the form (cf. [34])

$$
K_{e, s}\left(\overrightarrow{q_{1}}, \overrightarrow{q_{2}}\right)=\frac{8 \alpha^{2}}{\pi} \int_{0}^{1} d x \int_{0}^{1} d y \varphi_{e, s}\left(\overrightarrow{q_{1}}, \overrightarrow{q_{2}} ; x, y\right)
$$

where for the electrons

$$
\begin{gathered}
\varphi_{e}\left(\overrightarrow{q_{1}}, \overrightarrow{q_{2}} ; x, y\right)= \\
\frac{(x(1-x)+x(1-y)-4 x(1-x) y(1-y))\left|q_{1}\right|^{2}\left|q_{2}\right|^{2}-2 x(1-x) y(1-y)\left(\overrightarrow{q_{1}}, \overrightarrow{q_{2}}\right)^{2}}{x(1-x)\left|q_{1}\right|^{2}+y(1-y)\left|q_{2}\right|^{2}}
\end{gathered}
$$

and for scalar particles

$$
\begin{gathered}
\varphi_{s}\left(\overrightarrow{q_{1}}, \overrightarrow{q_{2}} ; x, y\right)= \\
\frac{(x(1-x)+x(1-y)-8 x(1-x) y(1-y))\left|q_{1}\right|^{2}\left|q_{2}\right|^{2}+4 x(1-x) y(1-y)\left(\overrightarrow{q_{1}}, \overrightarrow{q_{2}}\right)^{2}}{4\left(x(1-x)\left|q_{1}\right|^{2}+y(1-y)\left|q_{2}\right|^{2}\right)} .
\end{gathered}
$$

Note, that for the supersymmetric QED, where there are scalar charged particles, the total contribution to the kernel is

$$
\frac{\varphi_{e}\left(\overrightarrow{q_{1}}, \overrightarrow{q_{2}} ; x, y\right)+2 \varphi_{s}\left(\overrightarrow{q_{1}}, \overrightarrow{q_{2}} ; x, y\right)}{\left|q_{1}\right|^{2}\left|q_{2}\right|^{2}}=\frac{3 x(1-x)+3 x(1-y)-16 x(1-x) y(1-y)}{2\left(x(1-x)\left|q_{1}\right|^{2}+y(1-y)\left|q_{2}\right|^{2}\right)}
$$

and does not contain the term proportional to $\left(\overrightarrow{q_{1}}, \overrightarrow{q_{2}}\right)^{2}$.

The total cross-section in LLA can be written in the form

$$
\sigma_{t o t}=\int_{a-i \infty}^{a+i \infty} \frac{d \omega}{2 \pi}\left(\frac{s}{m^{2}}\right)^{\omega} \int \frac{d^{2} q_{1} \Phi_{A}\left(q_{1}\right)}{(2 \pi)^{2}\left|q_{1}\right|^{2}} \frac{d^{2} q_{1} \Phi_{B}\left(q_{2}\right)}{(2 \pi)^{2}\left|q_{2}\right|^{2}} f_{\omega}\left(\overrightarrow{q_{1}}, \overrightarrow{q_{2}}\right)
$$


where the $t$-channel partial wave $f_{\omega}\left(\overrightarrow{q_{1}}, \overrightarrow{q_{2}}\right)$ for the $\gamma^{*} \gamma^{*}$-scattering satisfies the BetheSalpeter equation

$$
\omega f_{\omega}\left(\overrightarrow{q_{1}}, \overrightarrow{q_{2}}\right)=(2 \pi)^{2} \delta^{2}\left(q-q^{\prime}\right)+\int \frac{d^{2} q_{1}^{\prime}}{(2 \pi)^{2}\left|q_{1}\right|^{2}\left|q_{1}^{\prime}\right|^{2}} K\left(\overrightarrow{q_{1}}, \overrightarrow{q_{1}^{\prime}}\right) f_{\omega}\left(\overrightarrow{q_{1}^{\prime}}, \overrightarrow{q_{2}}\right) .
$$

The solution of this equation can be obtained in the form of the Fourier expansion

$$
f_{\omega}\left(\overrightarrow{q_{1}}, \overrightarrow{q_{2}}\right)=\sum_{n=-\infty}^{\infty} \int_{-\infty}^{\infty} d \nu e^{i n\left(\varphi_{1}-\varphi_{2}\right)} \frac{2 \pi}{\left|q_{1}\right|\left|q_{2}\right|}\left(\frac{\left|q_{1}\right|}{\left|q_{2}\right|}\right)^{2 i \nu} \frac{1}{\omega-\omega(n, \nu)}
$$

where $\varphi_{k}$ is the phase of the two-dimensional vector $\overrightarrow{q_{k}}$ written in the complex form as $\left|q_{k}\right| e^{i \varphi_{k}}$ and $\omega(n, \nu)$ is the eigenvalue of the homogeneous Bethe-Salpeter equation:

$$
\omega(n, \nu)=\int \frac{d^{2} q^{\prime}}{(2 \pi)^{2}|q|^{4}} K\left(\vec{q}, \overrightarrow{q^{\prime}}\right) e^{i n\left(\varphi^{\prime}-\varphi\right)}\left(\frac{\left|q^{\prime}\right|}{|q|}\right)^{2 i \nu-3} .
$$

Using the above expressions for $K_{e, s}\left(\vec{q}, \overrightarrow{q^{\prime}}\right)$, it is easy to calculate $\omega(n, \nu)$ for the spinor and scalar electrodynamics (cf. [34])

$$
\begin{aligned}
& \omega_{Q E D}(n, \nu)=\frac{\alpha^{2}}{32} \frac{\sinh (\pi \nu)}{(\cosh (\pi \nu))^{2}} \frac{1}{1+\nu^{2}} \frac{1}{\nu}\left(\left(11+12 \nu^{2}\right) \delta_{n}^{0}-\frac{1+4 \nu^{2}}{2} \delta_{|n|}^{2}\right), \\
& \omega_{S E D}(n, \nu)=\frac{\alpha^{2}}{64} \frac{\sinh (\pi \nu)}{(\cosh (\pi \nu))^{2}} \frac{1}{1+\nu^{2}} \frac{1}{\nu}\left(\left(5+4 \nu^{2}\right) \delta_{n}^{0}+\frac{1+4 \nu^{2}}{2} \delta_{|n|}^{2}\right) .
\end{aligned}
$$

The high energy behaviour of the total cross-section is

$$
\sigma_{t o t} \sim \sum_{n=0}^{\infty} c_{n} s^{\Delta(n)} \cos \left(n\left(\varphi^{\prime}-\varphi\right)\right)
$$

where $\Delta$ is the Pomeron intercept:

$$
\begin{aligned}
\Delta_{Q E D}(n) & =\pi \alpha^{2}\left(\frac{11}{32} \delta_{n}^{0}-\frac{1}{64} \delta_{n}^{2}\right) \\
\Delta_{S E D}(n) & =\pi \alpha^{2}\left(\frac{5}{64} \delta_{n}^{0}+\frac{1}{128} \delta_{n}^{2}\right) .
\end{aligned}
$$

Note, that for the supersymmetric QED the results are especially simple:

$$
\omega_{S U S Y}(n, \nu)=\frac{\alpha^{2}}{2 \nu} \frac{\sinh (\pi \nu)}{(\cosh (\pi \nu))^{2}} \delta_{n}^{0}, \Delta_{S U S Y}(n)=\frac{\pi \alpha^{2}}{2} \delta_{n}^{0} .
$$

\section{References}

[1] L.N. Lipatov, Sov. J. Nucl. Phys. 23 (1976) 642;

E.A. Kuraev, L.N. Lipatov and V.S. Fadin, Phys. Lett. B60 (1975) 50; Sov. Phys. JETP 44 (1976) 45; 45 (1977) 199. 
[2] Ya.Ya. Balitsky and L.N. Lipatov, Sov. J. Nucl. Phys. 28 (1978) 822; Sov. Phys. JETP Lett. 30 (1979) 355.

[3] V.N. Gribov and L.N. Lipatov, Sov. J. Nucl. Phys. 18 (1972) 438, 675;

L.N. Lipatov, Sov. J. Nucl. Phys. 20 (1975) 93;

G. Altarelli and G. Parisi, Nucl. Phys. B126 (1977) 298;

Yu.L. Dokshitzer, Sov. Phys. JETP 46 (1977) 641.

[4] H1 Collaboration, S. Aid et al., Nucl. Phys. B470 (1996) 3;

ZEUS Collaboration, M. Derrick et al., Zeit. Phys. C69 (1996) 607.

[5] G. Gurci, W. Furmanski and R. Petronzio, Nucl. Phys. B175 (1980) 27;

W. Furmanski and R. Petronzio, Phys. Lett. B97 (1980) 437.

[6] L.N. Lipatov and V.S. Fadin, Sov. J. Nucl. Phys. 50 (1989) 712.

[7] V.S. Fadin and L.N. Lipatov, Phys. Lett. B429 (1998) 127.

[8] G. Camici and M. Ciafaloni, Phys. Lett. B430 (1998) 349.

[9] S.J. Brodsky, V.S. Fadin, V.T. Kim, L.N. Lipatov and G.B. Pivovarov, JETP. Lett. 70 (1999) 155;

V.T. Kim, L.N. Lipatov and G.B. Pivovarov, IITAP-99-013, hep-ph/9911228; IITAP99-014, hep-ph/9911242.

[10] L3 Collaboration, M. Acciarri et al., Phys. Lett. B453 (1999) 94;

talk presented by M. Kienzle-Focacci at 8th Blois Workshop on Elastic and diffractive scattering (EDS99), Protvino, Russia, June 27 - July 2, 1999, to appear in the Proceedings.

[11] B. Andersson, G. Gustavson and J. Samuelson, Nucl. Phys. B467 (1996) 443;

B. Andersson, G. Gustavson and H. Kharraziha, Phys. Rev. D57 (1998) 5543;

G. Salam, JHEP 9807 (1998) 019;

M. Ciafaloni, D. Colferai and G.P. Salam, JHEP 9910 (1999) 017; Phys. Rev. D60 (1999) 114036;

M. Ciafaloni and D. Colferai, Phys. Lett. B452 (1999) 372;

R.S. Thorne, Phys. Rev. D60 (1999) 054031;

G. Altarelli, R.D. Ball and S. Forte, CERN-TH-99-317, hep-ph/9911273; CERN-TH2000-010, hep-ph/0001157.

[12] L.N. Lipatov, Sov. Phys. JETP 63 (1986) 904.

[13] L. N. Lipatov, Phys. Lett. B309 (1993) 394, preprint DFPD/93/TH/70, hepth/9311037.

[14] J. Bartels, Nucl. Phys. B175 (1980) 365;

J. Kwiecinski and M. Prascalowich, Phys. Lett. B94 (1980) 413.

[15] L. N. Lipatov, Sov. Phys. JETP Lett. 59 (1994) 571;

L. D. Faddeev and G. P. Korchemsky, Phys. Lett. B342 (1995) 311. 
[16] L. N. Lipatov, Nucl. Phys. B548 (1999) 328.

[17] L. N. Lipatov, Proceedings of the International Workshop DIS'99, Zeuthen, April 1999, pp. 207-209;

J. Bartels, G. P. Vacca and L. N. Lipatov, Phys. Lett. B477 (2000) 178.

[18] L.N. Lipatov, Nucl. Phys. B452 (1995) 369; Physics Reports 286 (1997) 132.

[19] A. P. Bukhvostov, G. V. Frolov, E. A. Kuraev and L. N. Lipatov, Nucl. Phys. B258 (1985) 601.

[20] L. N. Lipatov, Perspectives in Hadronic Physics, Proc. of the ICTP conf. (World Scientific, Singapore, 1997).

[21] J. Maldacena, Adv. Theor. Phys. 2 (1998) 231, Int. J. Theor. Phys. 38 (1998) 1113.

[22] V.S. Fadin, R. Fiore and M.I. Kotsky, Phys. Lett. B359 (1995) 181; B387 (1996) 593.

[23] V.S. Fadin and L.N. Lipatov, Nucl. Phys. B406 (1993) 259;

V.S. Fadin, R. Fiore and A. Quartarolo, Phys. Rev. D50 (1994) 5893;

V.S. Fadin, R. Fiore and M.I. Kotsky, Phys. Lett. B389 (1996) 737.

[24] V.S. Fadin and L.N. Lipatov, Nucl. Phys. B477 (1996) 767;

V.S. Fadin, M.I. Kotsky and L.N. Lipatov, Phys. Lett. B389 (1996) 737.

[25] S. Catani, M. Ciafaloni and F. Hautman, Phys. Lett. B242 (1990) 97; Nucl. Phys. B366 (1991) 135;

G. Camici and M. Ciafaloni, Phys. Lett. B386 (1996) 341; Nucl. Phys. B496 (1997) 305 ;

V.S. Fadin, R. Fiore, A. Flashi and M.I. Kotsky, Phys. Lett. B422 (1998) 287.

[26] L. Durand, P.M. Fishbane and L.M. Simmons, J. Math. Phys. 17 (1976) 1973.

[27] K.G. Chetyrkin, A.L. Kataev and F.V. Tkachov, Nucl. Phys. B174 (1980) 447;

D.I. Kazakov and A.V. Kotikov, Theor. Math. Phys. 73 (1987) 126; Nucl. Phys. B307 (1988) 791.

[28] M.J. Levine, E. Remiddi and R. Roskies, Phys. Rev. D20 (1979) 2068;

W. Celmaster and R.J. Gonzalves, Phys. Rev. D21 (1980) 3112;

A. Terrano, Phys. Lett. B93 (1980) 349;

B. Lampe and G. Kramer, Phys. Scr. 28 (1986) 585.

[29] A.V. Kotikov, Phys. Lett. B375 (1996) 240.

[30] S. Catani, G. Marchesini and B.R. Webber, Nucl. Phys. B366 (1991) 135;

Yu.L. Dokshitzer, V.A. Khoze and S.I. Troyan, Phys. Rev. D53 (1996) 89. 
[31] J.A.M. Vermaseren, Acta Phys. Polon. B29 (1998) 2599; Int. J. Mod. Phys. A14 (1999) 2037;

S. Moch and J.A.M. Vermaseren, NIKHEF-99-030, hep-ph/9912355; NIKHEF-99022, hep-ph/9909269.

[32] L.Lewin, Polylogarithms and Associated Functions (North Holland, Amsterdam, 1981).

[33] A.I. Davydychev and J.B. Tausk, Phys. Rev. D53 (1996) 7381;

J. Fleischer, M.Yu. Kalmykov and A.V. Kotikov, Phys. Lett. B462 (1999) 169;

J. Fleischer and M.Yu. Kalmykov, Phys. Lett. B470 (1999) 168;

A.I. Davydychev, Phys. Rev. D61 (2000) 087701.

[34] V. N. Gribov, L. N. Lipatov and G. V. Frolov, Phys. Lett. B31 (1970) 34; Sov. J. Nucl. Phys. 12 (1971) 543;

H. Cheng and T. T. Wu, Phys. Rev. D1 (1970) 2775;

H. Cheng and T. T. Wu, Expanding Protons: Scattering at High Energies (MIT press, Cambridge, Massachusetts, 1987).

[35] A. Devoto and D.W. Duke, Riv. Nuovo Cim. 7 (1984) 1.

[36] N. Nilsen, Nova Acta 90 (1909) 125;

K.S. Kolbig, J.A. Mignaco and E. Remiddi, BIT 10 (1970) 38; Nuovo Cim. A11 (1972) 824. 